\title{
IMAGING TECHNIQUES IN THE DIAGNOSIS OF PULMONARY HYPERTENSION
}

DOI: 10.36740/WLek202009111

\author{
Weronika Topyła ${ }^{1}$, Michał Tomaszewski' , Agnieszka Wojtkowska' ${ }^{1}$, Sylwia Łukasik' ${ }^{1}$, Andrzej Wysokiński' ${ }^{1}$, \\ Anna Serefko \\ 'DEPARTMENT OF CARDIOLOGY, MEDICAL UNIVERSITY OF LUBLIN, LUBLIN, POLAND \\ 2DEPARTMENT OF APPLIED AND SOCIAL PHARMACY, LABORATORY OF PRECLINICAL TESTING, MEDICAL UNIVERSITY OF LUBLIN, LUBLIN, POLAND
}

\begin{abstract}
Introduction: Pulmonary hypertension is defined as being a haemodynamic state, wherein the mean pulmonary artery pressure measured during right heart catheterization is equal or greater than $25 \mathrm{mmHg}$. As a result, right ventricular heart failure develops and clinical symptoms such as dyspnea, fatigue, weakness, angina and fainting occur. The aim: To highlight the role of imaging techniques in diagnostic process for pulmonary hypertension.

Review and Disscusion: The diagnosis of pulmonary hypertension is multistage and often requires a number of studies. Currently, imaging techniques play a significant role in the diagnostic algorithm for pulmonary hypertension due to the fact that they are non-invasive and readily available, and many of their parameters are closely related to pulmonary hemodynamics.

Conclusions: The diagnosis of pulmonary hypertension requires a multistep approach and a number of imaging studies. The suspicion of the disease is based on medical history, clinical symptoms and chest radiogram. Echocardiography plays a crucial role in pulmonary hypertension detection. A computed tomography and cardiac magnetic resonance are valuable methods in determining the cause of suspected or confirmed pulmonary hypertension.
\end{abstract}

KEY WORDS: pulmonary hypertension, imaging, chest radiogram, echocardiography, V/Q scan, computed tomography, cardiac magnetic resonance imaging

Wiad Lek. 2020;73(9 p. I):1867-1869

\section{INTRODUCTION}

Pulmonary hypertension $(\mathrm{PH})$ is a rare, progressive disease in which abnormal pressure in the pulmonary arteries increases due to pulmonary vascular, lung parenchyma or heart disease. The incidence and prevalence of $\mathrm{PH}$ in Europe varies between 15-60 cases per million population and 5-10 cases per million per year [1]. The symptoms of pulmonary hypertension are non-specific and mainly related to progressive right ventricular failure. Initially, they occur only during physical activity and include dyspnea, fatigue, weakness, chest pain and syncope [2].

Depending on the etiological factor, there are five main groups of pulmonary hypertension:

1) pulmonary arterial hypertension $(\mathrm{PAH})$,

2) pulmonary hypertension due to left heart disease,

3) pulmonary hypertension due to lung disease,

4) chronic thromboembolic pulmonary hypertension (CTEPH)

5) pulmonary hypertension due to unknown causes [3]. The diagnosis of $\mathrm{PH}$ is often problematic due to non-specific symptoms, especially in the early stages of the disease. The diagnosis is based on mean pulmonary artery pressure $(\mathrm{mPAP}) \geq 25 \mathrm{mmHg}$ registered by invasive haemodynamic measurement [3]. In case of $\mathrm{PH}$ suspicion, there are a number of easily accessible and non-invasive studies. Imaging tests currently play a valuable role in the diag- nostic algorithm for $\mathrm{PH}$. These include chest radiogram, echocardiography, perfusion or ventilation/perfusion (V/Q) scan, computed tomography or cardiac magnetic resonance imaging.

\section{THE AIM}

The aim of the review is to highlight the role of imaging techniques in diagnostic process for pulmonary hypertension.

\section{REVIEW}

\section{CHEST RADIOGRAM}

Chest X-ray (CXR) is often the first-line study performed in patients with cardio-vascular symptoms. It has relatively high sensitivity and specificity in detecting signs of pulmonary hypertension in the late stages [1]. For example, in $90 \%$ of idiopathic pulmonary arterial hypertension (IPAH) patients, the CXR is abnormal at the time of diagnosis [4]. The CXR abnormalities suggesting pulmonary hypertension include right ventricular (RV) and right atrial (RA) outline dilatation. The most characteristic abnormality is central main pulmonary artery (MPA) dilatation, contrasting with poor peripheral pulmonary vascular drawing, 
referred to as peripheral vessel amputation. In advanced $\mathrm{PH}$, pericardial effusion is observed, being visible in the CXR as a "waterflask" contour. The heart silhouette is then enlarged. Additionally, the lateral projection shows pericardial fluid and pericardial or mediastinal fat [5]. The presence of this symptom is associated with poor prognosis [6]. The CXR may be helpful in the first line differential diagnosis of $\mathrm{PH}$. Herein, pulmonary congestion may be a reason for pulmonary hypertension due to left heart disease, while parenchymal lesions and emphysema suggest $\mathrm{PH}$ due to lung disease such as interstitial lung diseases or chronic obstructive pulmonary disease (COPD) [3]. CXR may be useful also in distinguishing between arterial and venous $\mathrm{PH}$ by demonstrating the increase or decrease in the pulmonary vein-to-pulmonary artery ratio (PV/PA), respectively. The symptom suggesting $\mathrm{PAH}$ is increased $\mathrm{PV} / \mathrm{PA}$ ratio on CXR. The reduced index values suggest venous PH [7]. However, the normal CXR does not exclude $\mathrm{PH}$, especially in the early stages.

\section{ECHOCARDIOGRAPHY}

If $\mathrm{PH}$ is suspected, echocardiography should be performed first, as it is a non-invasive and readily available diagnostic tool. Many echocardiographic parameters are closely related to pulmonary haemodynamics. In patients with advanced $\mathrm{PH}$, right ventricle and atrium dilation, abnormal interventricular septal motion, right ventricular wall thickening and MPA dilation may be observed. However, one of the most relevant values is maximum tricuspid regurgitation wave velocity (TRVmax), where the value $>3.4 \mathrm{~m} / \mathrm{s}$ indicates high probability of diagnosis [8]. According to European Society of Cardiology (ESC) guidelines, in the assessment of $\mathrm{PH}$, the following echocardiographic parameters should be measured: right-to-left ventricle (RV/LV) diameter ratio, pulmonary artery acceleration time and right ventricular ejection time, peak and end-diastolic velocities of pulmonary regurgitant flow, main pulmonary artery diameter, collapsibility index and inspiratory diameter of the inferior vena cava (IVC), and the right atrial area [3]. Based on these measurements, echocardiographic probability of disease may be estimated. Echocardiographic outcome is crucial to decide whether cardiac catheterization is necessary.

In clinical practice, the systolic pulmonary artery pressure can be estimated with the use of the simplified Bernoulli equation. This is based on TRVmax and right atrial pressure (RAP) [9].

The RAP can be assessed based on collapsibility index and inspiratory diameter of IVC: the diameter of IVC $<2.1$ $\mathrm{cm}$, which collapses $>50 \%$ indicates normal RAP of about 0-5 $\mathrm{mm} \mathrm{Hg}$.

Recent reports highlight the significant role of tricuspid annular plane systolic excursion (TAPSE) as a strong prognostic factor in PAH patients. A normal value is 15 to 25 $\mathrm{mm}$. It has been established that TAPSE $\geq 2 \mathrm{~cm}$ strongly correlates with increased survival in these patients [10]. Echocardiography may also be helpful in determining the cause of suspected or confirmed PH.

\section{MULTISLICE COMPUTED TOMOGRAPHY}

Multislice computed tomography (MSCT) is helpful in specifying the cause of $\mathrm{PH}$, such as $\mathrm{CTEPH}$, lung disease, congenital heart disease (CHD) or esophageal dilatation in scleroderma, as well as in gaining prognostic information. Devaraj et al. demonstrated that the combined assessment of echocardiography and angio-CT enables better correlation of results with haemodynamic testing [11]. The features of pulmonary hypertension that may be easily diagnosed by computed tomography (CT) are RV and RA dilatation, MPA enlargement (>29 mm) and the increase of the MPA diameter to the ascending aorta (AA) diameter ratio above 1. Additionally, a broncho-arterial (BA) ratio $>1: 1$ in three or four lung lobes has a high specificity in the diagnosis of $\mathrm{PH}$ [12]. High Resolution Computed Tomography (HRCT) is particularly extremely helpful in the diagnosis of interstitial lung disease and emphysema, as abnormalities such as ground glass opacity with simultaneous interlobular septal thickening suggest pulmonary venoocclusive disease (PVOD) [13]. Additionally, angio-CT can be useful in CETPH diagnosis. Typical angiographic abnormalities in CETPH include total vascular occlusion, asymmetric filling defect, as well as the presence of organized thrombus with fibrosis [14]. Furthermore a CT pulmonary angiogram (CTPA) helps to determine whether the patient with CTEPH is eligible for surgical treatment.

\section{MAGNETIC RESONANCE}

In recent years, there have been many reports on cardiac magnetic resonance imaging (CMRI) being a valuable diagnostic tool in PH diagnosis. Compared to echocardiography, MRI images are not limited by acoustic windows, and, hence, show spatial resolution better. Due to high cost and limited availability, this study is not used to diagnose $\mathrm{PH}$ routinely. However, its usefulness for the assessment of right heart structure, volumes and function in patients with PAH [15] has been established. One of the abnormalities occurring in $\mathrm{PH}$ is RV muscle hypertrophy. In CMRI it is possible to estimate "a ventricular mass index" (RV mass divided by LV mass). When the value of this exceeds 0,6, it is significant for PH [16]. Similar to CT, a MPA diameter exceeding $29 \mathrm{~mm}$ or MPA/AA ratio $>1$ indicates pulmonary hypertension [17]. Moreover, the presence of late gadolinium enhancement, decreased PA dilatation and the presence of retrograde blood flow in PA have high predictive value for $\mathrm{PH}$ [18]. The CMRI may also be helpful in cases of suspected $\mathrm{CHD}$, if the echocardiographic images are not clear. This study can also be used as a radiation-free and safe method to assess both ventilation and perfusion in CTEPH [18].

\section{VENTILATION PERFUSION SCAN}

Ventilation/perfusion (V/Q) lung scan is the method of choice for CTEPH diagnosis. The normal V/Q scan or a scan indicating low probability for pulmonary embolism excludes CTPEH with $90-100 \%$ sensitivity and $94-100 \%$ specificity [20]. This diagnostic method reveals perfusion defects in areas with proper ventilation. A negative V/Q scan basically excludes CTEPH. A positive result (at least one or more segmental perfusion defect 
in an area of normal ventilation) is an indication for angio-CT [21]. The disadvantage of this exam is that these abnormalities are also visible in PVOD. The assessment of pulmonary parenchyma perfusion is a valuable parameter of postoperative evaluation of pulmonary vascular resistance (PVR) in CTEPH patients undergoing pulmonary endarterectomy (PEA).

\section{CONCLUSIONS}

There has been significant progress in the diagnosis of $\mathrm{PH}$ patients over the last three decades. This effect particularly concerns PAH. The diagnosis of $\mathrm{PH}$ requires a multistep approach and number of diagnostic studies. The initial suspicion of $\mathrm{PH}$ is established based on medical history, clinical symptoms, CXR and electrocardiogram. Current guidelines propose several new tools to facilitate $\mathrm{PH}$ diagnosis, especially more detailed rules for assessing the probability of $\mathrm{PH}$, using echocardiography. Particular echocardiographic parameters have been defined that should be taken into account in reaching such an assessment. CT and CMR play a significant role in the differential diagnosis of $\mathrm{PH}$ due to lung disease and/or hypoxia as these reveal features of interstitial lung disease, emphysema and PVOD. The angio- CT and V/Q lung scan, the last being the screening method of choice in the diagnosis of CTEPH, are particularly helpful in such situations. However, too low PH detection in the early stages remains a major problem, and limits or prevents the use of timely treatment that could improve long-term prognosis.

\section{REFERENCES}

1. Peacock AJ, Murphy NF, McMurray JJV, et al. An epidemiological study of pulmonary arterial hypertension. Eur Respir J. 2007;30:104-109.

2. Hoeper MM, Gibbs SR. The changing landscape of pulmonary arterial hypertension and implications for patient care. Eur Respir Rev. 2014;23:450-7.

3. Galiè N, Humbert M, Vachiery JL, et al. 2015 ESC/ERS Guidelines for the diagnosis and treatment of pulmonary hypertension. Eur Heart J. 2015;37(1):67-119.

4. Rich S, Dantzker DR, Ayres SM, et al. Primary pulmonary hypertension: a national prospective study. Ann Intern Med, 1987;107:216-223.

5. Ascha M, Renapurkar RD, Tonelli AR. A review of imaging modalities in pulmonary hypertension. Ann Thorac Med. 2017;12(2):61-73.

6. Grünig E, Peacock AJ. Imaging the heart in pulmonary hypertension: An update. Eur Respir Rev. 2015;24:653-64.

7. Milne EN. Forgotten gold in diagnosing pulmonary hypertension: the plain chest radiograph. Radiographics, 2012;32:1085-1087.

8. Kyranis SJ., Latona J, Platts D, et al. Improving the echocardiographic assessment of pulmonary pressure using the tricuspid regurgitant signalThe "chin" vs the"beard." Echocardiography, 2018;35(8):1085-1096.

9. Ensing G, Seward J, Darragh R, et al. Feasibility of generating hemodynamic pressure curves from noninvasive Doppler echocardiographic signals. J Am Coll Cardiol, 1994;23:434-442.

10. Mazurek JA, Vaidya A, Mathai SC, et al. Follow-up tricuspid annular plane systolic excursion predicts survival in pulmonary arterial hypertension. Pulmon Circul. 2017;7(2):361-371.

11. Devaraj A, Wells AU, Meister MG, et al. Detection of pulmonary hypertension with multidetector CT and echocardiography alone and in combination. Radiology 2010;254:609-616.
12. Shen $Y$, Wan C, Tian P, et al. CT-base pulmonary artery measurement in the detection of pulmonary hypertension: a meta-analysis and systematic review. Medicine (Baltimore) 2014;93:e256.

13. Montani D, Price LC, Dorfmuller P, et al. Pulmonary venoocclusive disease. Eur Respir J. 2009;33:189-200.

14. Dorfmüller $P$, Günther $S$, Ghigna MR, et al. Microvascular disease in chronic thromboembolic pulmonary hypertension: a role for pulmonary veins and systemic vasculature. Eur Respir J. 2014;44:1275-1288.

15. Mc Lure LER, Peacock AJ. Cardiac magnetic resonance imaging for the assessment of the heart and pulmonary circulation in pulmonary hypertension. Eur Respir J. 2009;33:1454-1466.

16. Peña E, Dennie C, Veinot J, Muñiz SH. Pulmonary hypertension: How the radiologist can help. Radiographics. 2012;32:9-32.

17. Swift AJ, Rajaram S, Marshall H, et al. Black blood MRI has diagnostic and prognostic value in the assessment of patients with pulmonary hypertension. Eur Radiol. 2012;22:695-702.

18. Oudiz RJ. Pulmonary hypertension associated with left-sided heart disease. Clin Chest Med. 2007;28:233-241.

19. Le Pavec J, Lorillon G, Jaïs X, et al. Pulmonary Langerhans cell histiocytosis-associated pulmonary hypertension: clinical characteristics and impact of pulmonary arterial hypertension therapies. Chest. 2012;142:1150-1157.

20. Lang IM, Plank C, Sadushi-Kolici R, et al. Imaging in pulmonary hypertension. JACC Cardiovasc Imaging. 2010;3:1287-95.

21. Bonderman D, Lang I. Chronic thromboembolic pulmonary hypertension. Eur Respir Monogr. 2012;57:108-118.

\section{ORCID and contributionship}

Weronika Topyła 0000-0002-3997-9952 A,B,D

Michał Tomaszewski 0000-0001-6993-2154 A,D,E,F

Agnieszka Wojtkowska 0000-0001-9219-9763 ${ }^{A, D}$

Sylwia Łukasik 0000-0002-1071-7262 B,D

Andrzej Wysokiński 0000-0002-4562-9705 A,E

Anna Serefko 0000-0002-5732-8950 D

\section{Conflict of interest}

Authors declare no conflict of interest

\author{
CORRESPONDING AUTHOR \\ Michał Tomaszewski \\ Department of Cardiology \\ Medical University of Lublin \\ Jaczewskiego 8, 20-954 Lublin, Poland \\ tel: $+48-81-7244151$ \\ e-mail: tomaszewskimd@gmail.com
}

Received: 06.07 .2020

Accepted: 07.09.2020

A - Work concept and design, B - Data collection and analysis, $\mathbf{C}$ - Responsibility for statistical analysis, 